Ophthalmologica 1972;164:465-472

\title{
Index rerum ad Vol. 164
}

\section{Confecit: C. Loeb-Schürch}

$(\mathrm{V})=$ Report - Vortrag - Communication $(\mathrm{B})=$ Book Reviews - Buchbesprechungen - Livres nouveaux

Ablatio retinae, v. Rotation Acide cétonique, v. Keto acid Adaptation à l'obscurité, v.

Indomethacin Aderhaut, v. Fluorescence angiography,

Kolobom Albinism; remarkable facts in human

albinism and leukism, 157 (B) Amblyopie, v. Congenital cataract,

Esotropies infantiles Amotio retinae, 156 (B) Amyloidosis, v. Lattice dystrophy Anaesthesie in extremen Altersklassen,

154 (B) Angiographie, v. Binocular angiography,

Drusen, Fluorescence angiography,

Haemangioma Angle camérulaire, v. Cyclodialysis,

Iridoschisis Angle closure, v. Iridoschisis Artificial lens; the introduction of an

artificial lens into the eye using

Binkhorst's technique, 387 (V) Astigmatismus, v. Keratoplasty Atlas of external diseases of the eye,

78 (B) Augenbinnendruck, v. Cyclodialysis,

Durchblutung, Kataraktextraktion Augenhintergrund; der Augenhinter-

grund, 149 (B) Augenmuskeln, v. Neuromyotonie,

Oculomotor paresis

Augenspiegelkurs. Untersuchungstechnik und Befunde, 408 (B)

Battement de paupières, v. Lacrimal

drainage Bing's local diagnosis in neurological

diseases, 151 (B) Binocular angiography; binocular fundus

fluorescence angiography, 398 (V) Biochemie, v. Keto acid, Nuclear

cataracts Biomicroscopie et histopathologie de

l'ceil, 156 (B) Blau-Gelb-Störung, v. Tritan plate Blinking, v. Lacrimal drainage Blocage de Tangle, v. Iridoschisis Blue-Yellow defect, v. Tritan plate Book reviews, 78 (B), 149 (B), 408

(B) Bovidés, v. Phospholine iodée Buchbesprechungen, 78 (B), 149 (B),

408 (B), 464 (B)

Canaliculi lacrimales, v. Lacrimal

drainage Cancer, v. Multiple tumors Capillary strip, v. Cyclodialysis Cataract, v. Cataractes congénitales, Congenital cataract, Kataraktextraktion, Kolobom, Nuclear cataracts, Phospholine iodée

466

Index rerum ad Vol. 164

Cataractes congénitales. Résultats opára-toires et etudes statistiques sur une période de plus de 25 ans (de 1944 au debut 1971), 422 
Cattle, v. Phospholine iodée

Cellular metabolism, v. Lattice dystrophy, Macular dystrophy

Central scotoma, v. Complamina

Chamber angle, v. Cyclodialysis, Irido-schisis

Champ visuel, v. Complamina, Drusen

Chien, v. Kristallartige Einschlüsse

Chirurgie, v. Artificial lens, Cataractes congénitales, Cyclodialysis, Katarakt-extraktion,

Keratoplasty, Kolobom, Rotation, Rotor-Instrument

Chorioidea, v. Fluorescence angiography, Kolobom

Chromatic information, v. Visual stimuli

Ciliary body, v. Fuchssches Epitheliom

Clinicopathologic case reports, 463

Collagen, v. Lattice dystrophy, Macular dystrophy

Color-vision, v. Indomethacin, Tritan plate

Complamina in macular degeneration. A blind study in central fields, 219

Cone vision; scotopic and photopic cone vision, 356 (V)

Congenital cataract and lens implantation, 392 (V)

Congrès, v. Netherlands Ophthalmo-logical Society

Conjunctivitis follicularis, v. Keto acid

Contact lens, v. Keratoplasty

Cornea; the cornea, 152 (B)

Cornea, v. Dysgenesis mesodermalis corneae, Fleckförmige Hornhaut-dystrophie, Indomethacin, Keratoplasty, Lattice dystrophy, Macular dystrophy

Corneal and external diseases of the eye, 78 (B)

Corps ciliaire, v. Fuchssches Epitheliom

Correspondance retinienne, v. Esotropies infantiles

Cortical cataract, v. Nuclear cataracts

Cosmic rays, v. Kosmische Strahlung Cristallin, v. Artificial lens, Cataractes congénitales, Congenital cataract, Kataraktextraktion, Nuclear cataracts, Phospholine iodée Cryoapplication, v. Makrophagen Cyclodialysis with hydroxyethyl metha-crylate capillary strip (HCS). Animal experiments with a new approach in glaucoma drainage surgery, 113

Deafness, v. Dysostose mandibulo-faciale Déchirure en fer à cheval, v. Horseshoe

tear Déchirure retinienne, v. Horseshoe tear,

Rotation Dégénérescence maculaire, v. Complamina, Depth localisation, Netzhaut-zysten

Dégénérescence retinienne, $v$. Netzhaut-

zysten, Retinazysten Depth localisation of pathological

processes in the retina, 375 (V) Désinsertion retinienne, v. Rotation Diagnostic différentiel, v. Neuromyotonie Dog, v. Kristallartige Einschlüsse Doppelbilder, v. Neuromyotonie Double vision, v. Neuromyotonie Drainage lacrymal, v. Lacrimal drainage Drusen; fluorescence angiography in circulatory disturbances in drusen of the optic disc, 449 Drusen, v. Dysostose mandibulo-faciale,

Netzhautzysten Dunkeladaptation, v. Indomethacin Durchblutung; Untersuchungen über die DurchblutunginNetzhautkapillarenbei intraokularen Drucksteigerungen, 249 Dysgenesis mesodermalis corneae; histologische und ätiologische Aspekte bei Dysgenesis mesodermalis corneae mit sekundärer Dysgenesis mesodermalis iridis, 182 Dysostose mandibulo-faciale avec difformité de Sprengel, naevus verruqueux de la face et anomalies du fond de Гceil, 97 
Index rerum ad Vol. 164

467

Dystrophie corneenne grillagee, v. Lattice

dystrophy Dystrophie corneenne maculaire,

v. Fleckförmige Hornhautdystrophie,

Macular dystrophy Dystrophies rétiniennes héréditaires,

v. Depth localisation

Ectasie corneenne, v. Keratoplasty

EEG, v. Visual stimuli

Effets secondaires, v. Indomethacin

Electromyogram, v. Neuromyotonie

Electrooculogram, v. Depth localisation, Indomethacin, Retinazysten

Electroretinogram, v. Depth localisation, Indomethacin, Retinazystem

Elektronenmikroskopie, v. Fleckförmige Hornhautdystrophie, Kristallartige Einschlüsse,

Makrophagen, Xanthom

EMG, v. Neuromyotonie

Endothelium; the endothelium of the cornea and its clinical implications, 79 (B)

Enzym, v. Keto acid

EOG, v. Depth localisation, Indomethacin, Retinazysten

Epithéliome de Fuchs, v. Fuchssches Epitheliom

Epithelium pigmentaire, v. Depth localisation, Fuchssches Epitheliom, Indomethacin,

Makrophagen

ERG, v. Depth localisation, Indomethacin, Retinazysten

Esotropies infantiles; age d'apparition des esotropies infantiles. Le pronostic des strabismes précoces, 208

Experimentelle Forschung, v. Cyclo-dialysis, Keto acid, Lacrimal drainage, Nuclear cataracts, Phospholine iodée, Rotor-Instrument

Eye symptoms in brain tumors, 464 (B)

Farbsinn, v. Indomethacin, Tritan plate Festschrift Hans Sautter zum 60. Ge-

burtstag, 241-335 Fleckförmige Dystrophie der Hornhaut,

v. Fleckförmige Hornhautdystrophie,

Macular dystrophy

Fleckförmige Hornhautdystrophie; licht-und elektronenmikroskopische Unter-suchungen über

die fleckförmige Hornhautdystrophie, 35

Fluorescence angiography in intraocular tumours, 161

Fluoroangiographie, v. Binocular angiography, Drusen, Fluorescence angiography,

Haemangioma

Fonctions visuelles, v. Cone vision, Visual stimuli

Fractures of the orbit, 152 (B)

Franceschetti-Zwahlen Syndrom, v. Dysostose mandibulo-faciale

Fuchssches Epitheliom; Beitrag zum benignen Epitheliom (Fuchs) des Ziliarkörpers, 321

Fundus camera, v. Binocular angiography

Gefässe, v. Drusen, Durchblutung,

Fluorescence angiography,

Haemangioma Genetics, v. Cataractes congénitales, 
Depth localisation, Dysgenesis

rnesodermalis corneae, Dysostose

mandibulo-faciale, Lattice dystrophy,

Macular dystrophy, Netzhautzysten Gesichtsfeld, v. Complamina, Drusen Gesichtssinn, v. Cone

vision, Visual

stimuli Gittrige Hornhautdegeneration,

v. Lattice dystrophy Glande lacrymale, v. Kristallartige

Einschlüsse Glaucoma, v. Cyclodialysis, Iridoschisis,

Kataraktextraktion, Phospholine iodée Glykoproteide, v. Fuchssches Epitheliom Gullstrandsches

Normalauge; ein Modell

für das Gullstrandsche Normalauge,

143

Haemangioma chorioideae, v. Fluorescence angiography

Haemangioma of the optic disc, 362 (V)

Haemangiomatosis retinae, v. Haemangioma

Heavy primaries, v. Kosmische Strahlung

468

Index rerum ad Vol. 164

Hereditary retinal dystrophy, v. Depth

localisation Hérédité, v. Cataractes congénitales, Depth localisation, Dysgenesis mesodermalis corneae, Dysostose mandi-bulo-faciale, Lattice dystrophy, Macular dystrophy, Netzhautzysten Histochemie, v. Fuchssches Epitheliom Histologie, v. Cyclodialysis, Dyegenesis mesodermalis corneae, Fleckförmige Hornhautdystrophie, Fluorescence angiography, Fuchssches Epitheliom, Iridoschisis, Lattice dystrophy, Macular dystrophy, Makrophagen, Xanthom Hornhaut, v.

Dysgenesis mesodermalis corneae, Fleckförmige Hornhautdystrophie, Indomethacin,

Keratoplasty, Lattice dystrophy, Macular dystrophy Horseshoe tear; the anatomy of the horseshoe tear, 361 (V) Hufeisenriss, v. Horseshoe tear Hund, v. Kristallartige Einschlüsse

Hydroxyethyl methacrylate, v. Cyclodialysis

Indocid, v. Indomethacin

Indomethacin; ocular side effects of indomethacin, 339

Indomethacin; retinopathy due to indomethacin, 385 (V)

Instrument, v. Artificial lens, Rotor-Instrument

Intraocular pressure, v. Cyclodialysis, Durchblutung, Kataraktextraktion

Iridoschisis and angle-closure glaucoma, 199

Iris, v. Dysgenesis mesodermalis corneae, Iridoschisis, Xanthom

Iris clip lens, v. Artificial lens, Congenital cataract

Kälteeinwirkung, v. Makrophagen Kammerwinkel, v. Cyclodialysis,

Iridoschisis Kaninchen, v. Cyclodialysis

Katarakt, v. Cataractes congénitales. Congenital cataract, Kataraktextraktion, Kolobom, Nuclear cataracts, Phospholine iodée

Kataraktextraktion; über die Kataraktextraktion bei Patienten mit prim'ár chronischem Glaukom, 284

Keratoconus, v. Keratoplasty

Keratom, v. Rotor-Instrument

Keratoplastik; das verätzte Auge und die Keratoplastik, 149 (B) 
Keratoplasty; lamellar keratoplasty in corneal ectasias, 50

Keratozyten, v. Fleckförmige Hornhautdystrophie, Lattice dystrophy, Macular dystrophy

Keto acid; on the keío acid content of tears, 71

Kollagen, v. Lattice dystrophy, Macular dystrophy

Kolobom; zur Operation der senilen Katarakt bei Augen mit angeborenem Kolobom der Uvea, 264

Kongress, v. Netherlands Ophthalmolo-gical Society

Kontaktlinse, v. Keratoplasty

Kosmische Strahlung; physiologische Wirkung der kosmischen Strahlung, 337

Krebs, v. Multiple tumors

Kristallartige Einschlüsse in den Zellen der Tränendrüse beim Hunde. Elek-

tronenmikroskopischeUntersuchungen, 122

Kryoapplikation, v. Makrophagen

Kyste, v. Netzhautzysten, Retinazysten

Lacrimal drainage; on lacrimal drainage, 409

Lacrimal gland, v. Kristallartige Einschlüsse

Lacrimal system; the lacrimal system, 464 (B)

Lähmung, v. Oculomotor paresis

Lamelläre Keratoplastik, v. Keratoplasty

Lapin, v. Cyclodialysis

Larmes, v. Keto acid

Index rerum ad Vol. 164

469

Lattice dystrophy; light microscopical and polarisation optical study of the lattice dystrophy of the cornea, 1 Lens, v. Artificial lens, Cataractes con-génitales, Congenital cataract, Kataraktextraktion, Nuclear cataracts, Phospholine iodée Lentille de contact, v. Keratoplasty Leukozyten, v. Durchblutung Lidschlag, v. Lacrimal drainage Linse, v. Artificial lens, Cataractes congénitales, Congenital cataract, Kataraktextraktion, Nuclear cataracts, Phospholine iodée Livres nouveaux, 78 (B), 149 (B), 408 (B) Lokalisation, v. Depth localisation

Maculadegeneration, v. Complamina, Depth localisation, Netzhautzysten

Macular dystrophy; light microscopical and polarisation optical study of the macular dystrophy of the cornea, 19

Makrophagen; zur Frage der Herkunft der Makrophagen nach Kälteeinwir-kung auf die menschliche Netzhaut. Eine elektronenmikroskopische Studie, 306

Malformation, v. Cataractes congénitales, Dysgenesis mesodermalis corneae, Dysostose mandibulo-faciale, Kolobom

Management of the patient with subnormal vision, 155 (B)

Mandibulo-faziale Dysostose, v. Dysostose mandibulo-faciale

Medikament, v. Complamina, Indo-methacin

Melanoma, v. Fluorescence angiography

Membrane épipapillaire, v. Dysostose mandibulo-faciale

Métabolisme céllulaire, v. Lattice dystrophy, Macular dystrophy

Methode, v. Artificial lens, Binocular angiography, Cyclodialysis, Depth localisation,

Keratoplasty, Refiecto-metry, Rotation

Mícrocirculation; advances in micro-circulation, 153 (B) 
Microscopie électronique, v. Fleck-förmige Hornhautdystrophie, Kristall-artige Einschlüsse, Makrophagen, Xanthom

Mikropapille, v. Dysostose mandibulo-faciale

Missbildung, v. Cataractes congénitales, Dysgenesis mesodermalis corneae, Dysostose mandibulo-faciale, Kolobom

Modell, v. Gullstrandsches Normalauge

Mucopolysaccharide, v. Fleckförmige Hornhautdystrophie, Fuchssches Epitheliom, Macular dystrophy

Müllersche Stützfasern, v. Depth localisation

Multiple tumors in ophthalmology, 81

Muscles oculaires, v. Neuromyotonie, Oculomotor paresis

Myotonie, v. Neuromyotonie

Naevus pigmentosus chorioideae, v. Fluorescence angiography

Naevus verruqueux hyperkératotique, v. Dysostose mandibulo-faciale

Nebenwirkungen, v. Indomethacin

Netherlands Ophthalmological Society. 164th meeting, Groningen 1970, 354-407 (V)

Netzhaut, v. Cone vision, Depth localisation, Durchblutung, Haemangioma, Horseshoe tear, Indomethacin, Makrophagen, Netzhautzysten, Retina-zysten

Netzhautdegeneration, v. Netzhautzysten, Retinazysten

Netzhautdystrophien, erbliche, v. Depth localisation

Netzhautzysten; über die Spätform der multiplen vitelliformen Netzhautzysten, 84

Neuromyotonie; zur Differentialdiagnose der okulären Neuromyotonie, 252

Neuro-Ophthalmologie, v. Neuromyotonie, Oculomotor paresis

Normal eye, v. Gullstransches Normalauge

470

Index rerum ad Vol. 164

Nuclear cataracts; some biochemical differences between cortical (pale) and nuclear (brown) cataracts, 228

Nucléotides, v. Phospholine iodée

Ocular examination, 158 (B)

Ocular muscles, v. Neuromyotonie, Oculomotor paresis

Oculomotor paresis; a case of oculomotor paresis, 405 (V)

Oculoplastic surgery, 154 (B)

Oedema papillae, v. Drusen

Ommatidia, v. Cone vision

Operation, v. Artificial lens, Cataractes congénitales, Cyclodialysis, Katarakt-extraktion,

Keratoplasty, Kolobom, Rotation, Rotor-Instrument

Ophthalmology, 408 (B)

Optic disc, v. Drusen, Dysostose mandibulo-faciale, Haemangioma

Oraabriss, v. Rotation

:î2P, v. Phospholine iodée Paedo-Ophthalmologie, v. Congenital

cataract, Esotropies infantiles Palsy, v. Oculomotor paresis Papille, v. Drusen, Dysostose mandibulo-faciale, Haemangioma Paralysie, v. Oculomotor paresis Pathologie; allgemeine Pathologie,

152 (B) Perception des couleurs, v. Indomethacin, 
Tritan plate Petersche Defektbildung, v. Dysgenesis

mesodermalis corneae Pharmacology; ocular pharmacology,

408 (B) Phospholine iodée; influence de la

phospholine iodée sur les nucléotides

libres de cristallins en survie, 128 Photopic system, v. Cone vision Physiologische Grundlagen der Hellig-

keits- und Farbempfindungen, 156 (B) Pigmentepithel, v. Depth localisation,

Fuchssches Epitheliom, Indomethacin,

Makrophagen Pilocarpin, v. Phospholine iodée

Plastic and maxillofacial trauma

symposium, 151 (B) Polarisationsmikroskopie, v. Lattice

dystrophy, Macular dystrophy Postoperative Wundheilungsstörungen,

155 (B) Pression oculaire, v. Cyclodialysis,

Durchblutung, Kataraktextraktion Primäre, schwere, v. Kosmische Strahlung Proceedings, v.

Netherlands Ophthalmo-

logical Society Prognosc, v. Esotropies infantiles Protection of vision in children, 150 (B)

Protein, v. Macular dystrophy, Nuclear

cataracts

Rabbit, v. Cyclodialysis

Radiation cosmique, v. Kosmische Strahlung

Recent research on the retina, 149 (B)

Receptors, v. Cone vision

Recherches expérimentales, v. Cyclodialysis, Keto acid, Lacrimal drainage, Nuclear cataracts, Phospholine iodée, Rotor-Instrument

Red-Green disorders, v. Tritan plate

Reflectometry; fundus reflectometry, 402 (V)

Refraktion, v. Keratoplasty

Retina, v. Cone vision, Depth localisation, Durchblutung, Haemangioma, Horseshoe tear, Indomethacin, Makrophagen, Netzhautzysten, Retinazysten

Retinal degeneration, v. Netzhautzysten, Retinazysten

Retinal disinsertion, v. Rotation

Retinale Korrespondenz, v. Esotropies infantiles

Retinazysten; ERG and EOG bei der Spätform der multiplen vitelliformen Retinazysten des

hinteren Augenpols, 440

Retinoblastoma, v. Multiple tumors

Retinopathy, v. Indomethacin

Retinoschisis iuvenilis, v. Depth localisation

Index rerum ad Vol. 164

471

Riegersche Dysplasie, v. Dysgenesis mesodermalis corneae

Rind, v. Phospholine iodée

Riss, retinaler, v. Horseshoe tear, Rotation

Rotation; treatment of upper semicircular retinal disinsertion by surgical rotation of the eyeball, 236

Rot-Grün-Störungen, v. Tritan plate 
Rotor-Instrument; experimentelle Unter-suchungen und klinische Erfahrungen mit neuen RotorInsírumenten, 273

Rotor-Messer, v. Rotor-Instrument

Saccus lacrimalis, v. Lacrimal drainage Sautter; Hans Sautter zum 60. Geburts-

tag, 241-335 Schielen, v. Congenital cataract,

Esotropies infantiles Scotome central, v. Complamina Scotopic system, v. Cone vision Scritti in onore del Prof. Luigi Maggiore,

153 (B) Serindeaminase; v. Keto acid Side effects, v. Indomethacin Sinopsi di oculistica, 157 (B) Spongiosa; klinische und experimentelle

Untersuchungen über die Transplantation autoplastischer Spongiosa, 155 (B) SprengeГsche

Anomalie, v. Dysostose

mandibulo-faciale Squint; the conservative management

of squint, 158 (B) Squint, v. Congenital cataract, Esotropies

infantiles Strabismus; the surgical and nonsurgical

management of strabismus, 158 (B) Strabismus, v. Congenital cataract,

Esotropies infantiles Strahlentherapie; spezielle Strahlen-

therapie gutartiger Erkrankungen.

Radiation therapy of benign diseases,

153 (B) Surdité, v. Dysostose mandibulo-faciale Surgery, v. Artificial lens, Cataractes

congénitales, Cyclodialysis, Katarakt-

extraktion, Keratoplasty, Kolobom,

Rotation, Rotor-Instrument

Symposium on ocular pharmacology and

therapeutics, 149 (B) Syndrom, v. Dysostose mandibulo-faciale Syndromes en ophtalmologie, 150 (B) Synopsis; a synopsis of ophthalmology,

78 (B)

Taubheit, v. Dysostose mandibulo-faciale Tear, retinal, v. Horseshoe tear, Rotation Tears, v.

Keto acid Technik, v. Artificial lens, Binocular

angiography, Cyclodialysis, Depth

localisation, Keratoplasty, Reflecto-

metry, Rotation Tension oculaire, v. Cyclodialysis,

Durchblutung, Kataraktextraktion Textbook of 'the fundus of the eye',

159 (B) Therapie, v. Complamina, Esotropies

infantiles, Keratoplasty, Neuro-

myotonie, Rotation Tiefenlokalisation, v. Depth localisation Tränen, v. Keto acid Tränenabfluss, v. Lacrimal drainage Tränendrüse, v. Kristallartige Einschlüsse Transactions of the American Ophthalmological Society, 464 (B) Transplantation cornéenne, v. Keratoplasty Trepan, v. RotorInstrument Tritan plate; the Farnsworth Tritan plate,

137 Troubles bleu-jaune, v. Tritan plate Troubles rouge-vert, v. Tritan plate Tumeurs de Гceil et des annexes

oculaires, 158 (B) Tumor, v. Fluorescence angiography,

Fuchssches Epithelium, Haemangioma,

Multiple tumors, Xanthom

Uvea-Trabekel-System; über die Struktur

des Uvea-Trabekel-Systems, 151 (B) Uvéites et virus, 157 (B)

Vaisseaux, v. Drusen, Durchblutung, Fluorescence angiography, Haemangioma 
472

Index rerum ad Vol. 164

Varia, 80, 160

Vererbung, v. Cataractes congénitales, Depth localisation, Dysgenesis mesodermalis corneae, Dysostose mandibulo-faciale, Lattice dystrophy, Macular dystrophy, Netzhautzysten

Vessels, v. Drusen, Durchblutung, Fluorescence angiography, Haemangioma

Visual field, v. Complamina, Drusen

Visual function, v. Cone vision, Visual stimuli

Visual stimuli; the representation in the EEG of chromatic information in visual stimuli, 355 (V)

Vitreiform degeneration, v. Horseshoe

tear Von Hippel disease, v. Haemangioma

Winkelblock, v. Iridoschisis

Xanthom der Iris. Ein klinisch-patholo-

gischer Befundbericht, 293 Xantinol nicotinate, v. Complamina

Z. Zellstoffwechsel

v. Lattice dystrophy,

Macular dystrophy Zentralskotom

v. Complamina Ziliarkörper

v. Fuchssches Epitheliom Zyste

v. Netzhautzysten

Retinazysten 